\title{
ONTOLOGÍA RELACIONAL Y COSMOPRAXIS, DESDE LOS ANDES. VISITAR Y CONMEMORAR ENTRE FAMILIAS AYMARA
}

\author{
RELATIONAL ONTOLOGY AND COSMOPRAXIS, SEEN FROM THE ANDES. \\ VISITING AND COMMEMORATING AMONG AYMARA FAMILIES
}

\begin{abstract}
Koen De Munter ${ }^{1}$
Este texto invita a repensar la pregunta antropológica central sobre qué es la "vida social" a partir de la obra reciente del antropólogo británico Tim Ingold, quien propone liberarla de su antropocentrismo y concebir lo social como unas dinámicas y continuas líneas de vida que fluyen en medio de y en relación con los otros procesos o líneas de vida. Estas líneas corresponden unas con otras. En términos "sociales" (y ecológicos), los miembros de un grupo cultural determinado aprenden a estar atentos a esta ontología relacional a través de un constante proceso de co-participación en el que los más experimentados educan la atención de las y los aprendices y en el que todos se habilitan mutuamente. Este aprendizaje socioecológico -que implica tanto un crear como un "someterse" a los procesos de crecimiento del estando-vivo- se deja analizar en términos de una cosmopraxis, y no tanto de una cosmovisión. La exploración teórica se nutre y se ilustra con el ejemplo de las conmemoraciones de los muertos y en general de la praxis del "visitar" o tumpaña en familias aymara en la ciudad de El Alto, Bolivia.

Palabras claves: ontología relacional, cosmopraxis, aymara, correspondencias, atención.
\end{abstract}

This article is an invitation to rethink the central anthropological question about what is social life -and what it means to be human - in dialogue with Tim Ingold's recent work, when he conceives social life-liberated from anthropocentrism-as a flux of continuous and open-ended "lines of life". These lines of life evolve amongst and along other lines of life, and correspond with them, in a relational world ("the life of lines"). As for "human correspondence", all members of a group are engaged in a process of education of attention where they learn to be attentive to the general meshwork of correspondences through a process of co-participation: novices and more experienced members co-participate in socio-ecological enskilment through their everyday or ritual practices. In our analysis, cosmopraxis prevails over cosmovision and doing partakes of a dance of animacy, so that anthropogenesis should always be seen as a "making-in-the-growing". This theoretical reflection about the lines of social life is inspired and illustrated by the important example of the commemorations of the dead and the praxis of "visiting" or tumpaña amongst Aymara families in El Alto, Bolivia.

Key words: Relational ontology, cosmopraxis, Aymara, correspondences, attention.

What if we think of the earth's surface not as already laid out, only awaiting discovery and occupation, but rather as continually unfolding in the course of life itself, through the movements of people and animals, wind and currents, celestial bodies and so on? (Ingold 2010:168). Human beings are auto-fabricators, said Ortega. They make or build themselves. But humans also grow: like all living beings they undergo a process of ontogenesis (Ingold 2015:120).

\section{Vida Social y Ecología Sintiente}

En el presente texto exploramos un abordaje antropológico de la vida social lato sensu, analizando lo social como prácticas y dinámicas de coparticipación a través de las cuales los humanos aprenden a relacionarse y se relacionan o conviven, no solamente con otros humanos, sino con otros seres vivos e integrantes de la vida, y en general, adoptando una noción central en la obra reciente de Tim Ingold, con otras "líneas de vida". "La vida" se concibe aquí como el "estando-vivo", como diferentes líneas de vida que se desenvuelven continuamente, relacionadas entre sí, en medio de una "ecología sintiente" 1 -sensibilidades y orientaciones que se desarrollan a medida que los seres vivos van experimentando la vida a través de los movimientos, sonidos, etc., de los demás seres e integrantes del mundo y de ellos mismos. Es en este sentido muy

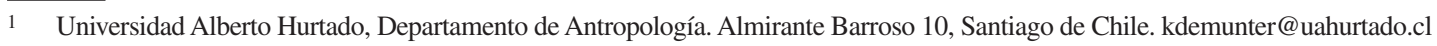


general -un proceso abierto de ser y estar a través de constantes relaciones entre diferentes líneas de vida- que concebimos aquí la noción de ontología relacional. La reflexión antropológica respecto del convivir, considerado en su plena dimensión relacional-ecológica, nos lleva, en la segunda mitad de este texto, a un comentario de un ejemplo concreto de prácticas sociales. Ahí se verá cómo, en la heterogénea y dinámica tradición aymara ${ }^{2}$ (Caqueo et al. 2014; De Munter 2007, 2016a), las familias visitan y conmemoran a los muertos y cómo, haciendo esto, se habilitan para relacionarse con los procesos de vida en general. Al mismo tiempo, esta experiencia antropológica particular con familias aymara inspira la teorización general del convivir en relación con el estando-vivo. Nuestro propósito principal es analizar desde una antropología de las prácticas, orientada a mirar la vida social directamente a partir de la praxis del convivir -y no a partir de representaciones simbólicas, esquemas cognitivos y estructuras o marcos organizativos subyacentes-cómo las personas conviven y aprenden a convivir a lo largo de esta ontología relacional. Si hablamos de "praxis del convivir" no solo un (aprender a) actuar "socialmente" como agencia intencionada, sino también un someterse a un (saber) dejarse llevar por el "estando-vivo" $\mathrm{y}$, haciendo el camino, aprender a relacionarse y sintonizarse, también con estas dinámicas de vida más amplias. Ingold, en reflexiones recientes, al respecto usa como interesante metáfora el aoristo, un modo verbal "intermedio" (del antiguo griego) que se mueve de manera no muy definida entre el modo pasivo y el activo -poéticamente, llega a hablar de un "pasivo" apasionado y atento (Ingold 2015)-. Se trata entonces de comprender de qué manera(s) se fomenta la "educación de la atención" (Ingold 2000, 2010) hacia cómo la socialidad 'humana' se entreteje con los procesos de vida en general, habilitando a los seres humanos en competencias sociales, lato sensu, que les permiten relacionarse con los otros integrantes del estando-vivo y que vuelven más atenta su disposición para ser educados por esta relacionalidad englobante (la educación, lato sensu, como dimensión central de lo social y cultural). Más que una ecología de la mente -recordando el conocido canon de Bateson- deberíamos entonces concebir una "ecología de la vida" (Ingold 2000:16).

En el primer apartado del artículo explicamos y contextualizamos algunos de los conceptos centrales para luego pasar, en el segundo, a presentar la noción de cosmopraxis y el rol principal de la educación y crianza. En el tercer y cuarto apartado nos enfocamos en una reflexión acerca de la importancia del visitar en el "practicar la familia" y en la praxis del conmemorar en la tradición aymara en particular.

\section{Ontología Relacional y Correspondencias}

Con ontología relacional nos referimos al "estando-vivo", que se caracteriza por las dinámicas relacionadoras que se producen entre sus integrantes (cosas, seres, entornos, ambientes). Como sugiere Tim Ingold, metafóricamente, podemos hablar de "trenzados" de vida, constantes y abiertos. Para pensar la socialidad humana como enlazada con los "trenzados" de vida en general, optamos por enfocar la experiencia, el aprendizaje hands-on (Reed 1999), la creatividad y la improvisación y nos alejamos de lecturas en clave de intencionalidad (Duranti 2015), de dominio previo -"conocimiento"- de estructuras, conceptos, reglas o normas sociales. Esta aproximación, distante de enfoques semióticos y cognitivos, se presenta como íntimamente involucrada (teóricamente, empíricamente, pedagógicamente) con los flujos mismos de la praxis social que se encuentran inmersos en el estando-vivo. Esto sigue una concepción de la antropología según la que esta, política y éticamente, siempre tendría que aspirar a ser una antropología con -un abrirse y responder a la vida - y no una antropología sobre -describir o representar el mundo de grupos particulares. It is to convert every certainty into a question, whose answer is to be found by attending to what lies before us, in the world, not by looking it up at the back of the book (Ingold 2013:2, énfasis mío). La antropología por la que aboga Ingold se propone investigar 'importantes preguntas' para la humanidad, como por ejemplo, de manera muy general, pero fundamental: ¿qué hace que determinadas relaciones de vida, desde la perspectiva de las prácticas humanas, se puedan considerar como sociales? Y por ende, "convirtiendo cada certidumbre en una pregunta" y desde la misma perspectiva, ¿qué sería lo social? Y también, ¿cómo se "aprende" esta "socialidad"? La antropología busca respuestas a estas preguntas a lo largo de un involucramiento afectivo y compromiso sostenido con determinados grupos humanos y con toda la realidad viviente con y en la que estos se desenvuelven -al igual que las y los antropólogos mismos. Así, sobre la base de tal compromiso antropológico (vivencial, 
reflexivo-teórico y etnográfico a la vez), fomentando una sobria y sostenida atención antropológica hacia las prácticas creadoras de y creadas por la socialidad humana, en el presente artículo nos interesa entender cómo esta praxis de la convivencia humana -la que presupone siempre un aprender y enseñar, un habilitarse- se entreteje inexorablemente con los 'procesos de vida' más amplios. Podríamos hablar, en términos muy generales, de una convivencia o socialidad ecológica, inspirados por Félix Guattari cuando habla de una ecología social y también del socius -el campo de lo social que cambia mediante pequeñas acciones cotidianas sociales-ecológicas que se auto-promueven (Guattari 1989)-. Según la perspectiva antropológica que adoptamos aquí, el epíteto "ecológico" de alguna manera resulta incluso ser redundante, ya que lo social se da siempre de manera inherentemente ecológica.

Cuando hablamos de socialidad -el concepto más genérico- o de convivencia, el enfoque no caerá entonces tanto en lo que se suele entender como sociedad (concíbase como colectivo o como suma de individuos), sino en cómo las personas y los grupos humanos viven juntándose y relacionándose, más allá de definiciones o circunscripciones antropocéntricas de familia o comunidad. Las vidas humanas se desenvuelven de manera fundamentalmente relacional, a través de continuas relaciones -en sintonía o conflicto- con los entornos y con otros integrantes del estando-vivo. Vivir es siempre un devenir en relación con otros, un dar forma y un ser formado (un "criar y dejarse criar", recordando los planteamientos andinos de PRATEC), lo que tiene importantes implicancias para cómo entender las relaciones entre humanos, animales y el medio ambiente (socialidad sensu lato) y para nociones antropológicamente tan centrales como habilidades y aprendizajes (Chaiklin y Lave 2001; Hastrup 2015; Ingold 2015; Lave 1991). Con miras a abrir aún más esta visión ampliada de la vida social como procesos de vida que se desenvuelven en medio de y a lo largo de la ontología relacional, en recientes publicaciones y discursos, Tim Ingold propone hablar de múltiples correspondencias entre las "líneas" producidas por las respectivas trayectorias y experiencias de vida (Ingold 2014, 2015). Estas líneas son como senderos (thakhi podría ser una sugestiva traducción en aymara) de crecimiento y movimiento que van "correspondiendo" con otros senderos de vida. Todos estos flujos de cor-respondencias van constituyendo la malla (meshwork) continua y abierta del 'estando-vivo' (Ingold 2010). Estas correspondencias se producen a medida que se van entretejiendo ("respondiendo") las líneas de vida trenzadas por las criaturas vivas entre-acudiendo a distinciones difusas y flexibles- las cosas y materiales "vibrantes" 4 (fibras, greda, hojas, etc.), los seres vivos (humanos, animales, plantas, agua, etc.), el cosmos (cielo, sol, luna, estrellas, etc.) y los integrantes de los 'paisajes' (wak'as, uywiris, chacras, etc., vistos desde contextos andinos). Si consideramos esta multitud de correspondencias específicamente desde la perspectiva de la socialidad o praxis social humana, se puede hablar de "correspondencia humana" (Ingold 2015).

En este limitado espacio no abordaremos directamente la importante discusión teórica sobre la 'agencia humana': efectivamente ¿cómo pensar la agencia si se deja de lado el paradigma de la intencionalidad y de la proyección cognitiva del mundo y cuando, más que de agencia, se prefiere hablar de "danza de animacidad" (dance of animacy, en Ingold 2010) o de un doing-in-undergoing (Ingold 2015)? Tampoco entramos aquí en el meta-debate reciente sobre el supuesto "giro ontológico" en antropología (Holbraad, Pedersen y Viveiros 2014) y en general sobre otras propuestas para "purgar la antropología de su antropocentrismo" (Descola 2014:269). Esto forma parte de otros momentos y espacios de reflexión. Este ensayo antropológico propone, en diálogo con aprendizajes etnográficos y guiado por la reflexión filosófica-antropológica de Ingold, entender determinadas prácticas sociales -in casu "practicar la familia" en contextos andinos mediante el visitar y conmemorar a los muertoscomo parte de dinámicas relacionadoras más amplias, que trascienden el campo de lo 'socialmeramente-humano'. Rompiendo de esta manera, al mismo tiempo, con la distinción limitante entre lo vivo y lo muerto.

Asimismo, ese texto invita a investigar por medio de qué prácticas y movimientos muy concretos, en los grupos con los que se ha trabajado, se "educa la atención" hacia las correspondencias que caracterizan estos múltiples 'trenzados' de vida (De Munter 2016b). En estos grupos, si los concebimos como comunidades de aprendizaje, dichos movimientos y prácticas se pueden considerar como educadores de la atención hacia la vital ontología relacional y hacia cómo participar de ella, tanto en sus dimensiones conocidas o experimentadas como desconocidas o por experimentar. Una buena ilustración, en el ámbito 
aymara, serían las relaciones culturales y sociales con los uywiri, conocidos generalmente como los cerros protectores de determinadas comunidades, y experimentados por los comuneros a veces directamente como "educadores" (Choque Churata 2009). Con estos uywiri, la gente aymara (jaqi) se relaciona durante ciertas visitas (rituales-sociales/ festivas) cuando los honran mediante ofrendas o 'pagos', como si fueran honorables parientes. Otra "práctica relacionadora" conocida sería el floreo, que se refiere a una práctica social-ecológica que permite atender de manera simultáneamente cotidiana, festiva y ritual las "correspondencias" que se dan entre las familias de los pastores y sus animales, en medio de un entorno-ambiente donde son de importancia vital los bofedales y las vertientes de agua o juturi (Mamani 2010). Aquí es el encontrarse entre personas y animales -como correspondencia entre sus respectivas líneas de vida- que les enseña a las personas, desde muy niños, la importancia de crear-criar cariño y compadrazgo con ellos, de ir a visitar en la mañana del floreo propiamente tal a los juturi o vertientes 5 (Pachaguaya 2008), $\mathrm{y}$, en general, de cuidar el "baile de la vida", con ellos y con el medio ambiente (Orye 2011). Este encuentro-correspondencia no se refiere a momentos determinados sino que se tiene que concebir como una línea continua. Así, los bofedales y las vertientes se cuidan continuamente. Asimismo, el floreo se prepara -se "trenza"- a lo largo del año, cuando, en las casas, las mujeres confeccionan los aretes y chimpus: 'flores' tejidas en lanas multicolores que recuerdan el kurmi o arcoíris (Mamani 2010).

No acudimos a estos ejemplos para caracterizar estas prácticas como "andinas" o como representativas de una particular "tradición aymara". Si se habla, en este texto, de "cosmopraxis aymara" o si aludimos a cierta "socialidad andina", no es tanto porque estos ejemplos sean característicos de una "cultura aymara" o "mundo andino" (lo que podría ser, en cierta medida, el caso), sino en primer lugar porque se producen en contextos geográficos claramente andinos y porque los grupos que promueven estas prácticas se auto-consideran como aymara. Para nuestros propósitos, no se trata tanto de estudiar el caso particular de, por ejemplo, la 'socialidad aymara' en sí, sino más bien de abrirnos a qué nos podría enseñar el estudio de -con- esta 'socialidad aymara' de una manera más general (filosóficaantropológica, si se quiere) acerca de cómo la convivencia humana se produce a lo largo de y en cor-respondencia con los demás procesos de vida. Esta mirada antropológica, que se mueve entre lo particular y lo universal, sí podría, eventualmente, indicar ciertas "otras" maneras de aprovechar y poner en práctica la universal potencialidad humana de sentirse-parte-de-un-conjunto-más-grande, en el sentido inmanente de esta expresión (Pinxten 2007; Pinxten y De Munter 2010). Maneras o "caminos" (ways, con la rica connotación que siempre tiene este concepto en inglés, relevante aquí -Ingold habla de wayfaring/hacerse camino) que quedaron tal vez demasiado 'invisibilizados', no solamente por medio de ciertos paradigmas dominantes en las ciencias sociales, sino también en las políticas nacionales cuando conciben 'la sociedad', 'lo social' y 'lo cultural' de una manera demasiado separada de los ambientes/entornos ecológicos, lo que permite y justifica relaciones de proyección y de dominio de una parte hacia o frente a la otra (Hastrup 2015).

Aspiramos a explorar un alcance de la socialidad humana más amplio y abierto, desde una perspectiva según la que el continuo flujo de correspondencias a lo largo de los procesos de vida prima por sobre las acotadas interacciones entre actores o integrantes demasiado separados, movidos por supuestos 'fines', 'finalidades' o proyecciones, en sistemas relativamente cerrados (Berkes 2012; Ingold 2015). En este sentido, la relevancia de estudiar con personas, familias y grupos que practican la vida social según contextos "andinos" trasciende el interés por una identidad cultural particular.

Antes de pasar al ejemplo etnográfico principal de este artículo, respecto del visitar y el conmemorar entre los aymara, es necesario ofrecer una breve discusión del uso del concepto de cosmopraxis, en lugar del más conocido término "cosmovisión" y relacionar esta discusión con las pistas teóricas propuestas por Ingold sobre la vida relacional.

\section{Cosmopraxis y "Educar la Atención"}

En anteriores publicaciones hemos argumentado que, por razones antropológicas-filosóficas y para fines heurísticos y epistemológicos, nos parece recomendable sustituir el concepto de "cosmovisión" -y por ende, los presupuestos y alcances explicativos, interpretativos y también ideológicos que conllevapor el de "cosmopraxis" (De Munter 2010; De Munter y Note 2009). Esto implica invertir las todavía frecuentes perspectivas cognitivistas y constructivistas según las cuales la cultura o la sociedad se construye 
-y por ende, se puede explicar- sobre todo a partir de un conjunto de conocimientos (estructuras, lógicas, organigramas), considerado casi cartográficamente como su cosmovisión - worldview en inglés ${ }^{6}$-. Nos situamos así, de modo muy general, en la línea de lo que se suele conocer como una 'antropología de las prácticas' (Chaiklin y Lave 2001; Ingold 1996, 2014; Ortner 2006), según la cual para aproximarse a "lo social" son las prácticas que priman sobre los significados (lo que, obviamente, incluye también el relatarse historias o sueños, por ejemplo). Este enfoque no es nuevo, aunque en lo que concierne específicamente a los estudios andinos, el paradigma cognitivista y semiotizante, presente también en lecturas constructivistas, es el que ha prevalecido, aun cuando se toman en cuenta plenamente los contextos sociales y políticos. Como enfoque es afín, en cierta medida, a la pista praxeológica propuesta por Pierre Bourdieu, quien levantó la dicotomía entre sujeto y objeto, a partir de prácticas y movimientos muy concretamente encarnados que gatillan dinámicas de internalización y externalización. Nuestra aproximación sin embargo no presupone un dispositivo tipo habitus, sino que parte de preocupaciones más antropológicas, con mucho más atención en los constantes aprendizajes a lo largo del tiempo, para la creatividad y la improvisación (y no tanto "tácticas" o "astucias", como en las "artes del hacer" que planteaba De Certeau y también, aunque de otro modo, Bourdieu). Sobre todo, el abordaje por el que abogamos asume cuestiones más directamente 'ecológicas': investigar desde un "compromiso ontológico" (Ingold 2013) cómo "co-responde" el ser/estar social con el ser/estar vivo en general. Así, en concordancia con la obra de Ingold, concebimos que la experiencia humana conlleva el impulso vital a través de nuestros cuerpos móviles y nuestras prácticas cotidianas, prácticas que habilitan, que crean y a lo largo de las cuales nos dejamos criar.

La experiencia humana se mueve constantemente, bajo sus propios impulsos, pero también movida (y con-movida) por los contextos o entornos -con otros seres, cosas, paisajes, etc.-, produciéndose continuas sintonizaciones entre todos estos movimientos (o lines) y energías de vida. No por casualidad, Ingold habla a veces de "sinestesia", generalmente como adjetivo, sin las connotaciones cognitivas y neurológicas, pero de manera directa: "sentir conjuntamente". Abogamos entonces por una exploración antropológica de una ontología relacional en la que las personas y las familias, a través de sus prácticas, son criadas por y contribuyen a (como un someterse activo y un actuar sometiéndose) una dinámica malla de relaciones de vida, un sinfín de correspondencias que se mueven y reacomodan constantemente (Gieser 2008; Ingold 2015; Ingold y Kurttali 2000; Lee e Ingold 2006). Esto implica que hay que dejar de lado el concepto clásico de la cosmovisión o worldview, cuya premisa sería un conjunto ideológico-teórico que permite interpretar el mundo y que orienta e incluso dirige las acciones, permitiendo mirarlo y en rigor 'experimentarlo' desde fuera, de la misma manera que un científico objetivizante-imparcial, desde una distancia tan segura que es fácil caer en la ilusión que ese mundo no sería afectado por su presencia. Siempre según el 'paradigma-worldview', analíticamente se pretende superar esta distancia transfiriendo algo de esa cosmovisión en artefactos materiales o en ciertas costumbres que van a figurar entonces como vehículos de significados, distribuyéndolos en las cabezas y en 'objetos inscritos'. Sin embargo, la mente no puede formular el mundo antes de cualquier experiencia o involucramiento/compromiso (engagement). Para Ingold y Orye, aprehender el mundo no es una cuestión de construcción -y menos de inscripciónsino fundamentalmente de engagement a partir de constantes "encarnaciones vivas". No se trata tanto de tener o hacerse una visión del mundo/cosmos, sino de asumir una visión en él (Orye 2011). De ahí que preferimos hablar de cosmopraxis. Ambiente y organismo (humano u otro) participan de un mismo proceso. No caben las dicotomías entre cultura y naturaleza o entre lo 'construido' y lo 'dado'. Concebir cosmovisión, siguiendo una orientación cognitivista todavía bastante frecuente, como una noción no relacional y negar esa matriz relacional de la participación en el mundo, presupone y generaliza una nefasta separación de percepción, acción y aprendizaje. Los seres humanos no cargan en sus cabezas un conjunto de representaciones y reglas disponibles para transmitir de manera independiente de su actuar concreto en el mundo y ligadas a algo así como una 'moral accountability'. Si, como se propone hacer aquí, dejamos prevalecer las prácticas culturales (el morar en el mundo), se puede decir que son más bien ellas las que generan constantemente las significaciones culturales o sociales y también los valores humanos asociados (Allerton 2011; Overing y Passes 2000; Yampara y Temple 2008). Esta inversión implica alejarse de explicaciones 
sobre la base de la intencionalidad o de conceptos sociológicos como una 'conciencia colectiva' o un habitus internalizado, ya que no permiten captar el flujo y la complejidad de la vida social, una vida social que siempre de nuevo tiene que ser realizada, en medio de una ontología relacional.

Visto así, involucrarse en este mundo relacional tiene que ver en primer lugar con dinámicos procesos de aprendizaje. En lugar de hablar de una comunidad epistémica (paradigma cosmovisión), cuando hablamos de cosmopraxis concebimos más bien una "comunidad de prácticas y practicantes" (Chaiklin y Lave 2001; Lave 1991). Un aprendiz o una aprendiza -supongamos una joven aymara, hecha abstracción de si vive, como casi siempre es el caso, en un contexto de tensiones interculturalesnunca 'adquiere' cultura como si esta se descargase de una fuente superior de la sociedad a su mente. Más bien está ocupada con un proceso que la antropóloga Jean Lave (1991), conocida por su reconceptualización del aprendizaje en términos de práctica y coparticipación, denominó "entender practicando": dentro del proceso-vida, los miembros más experimentados guían a los más jóvenes a lo largo de una continua "education of attention" (Ingold 2000, 2010, 2015), proceso en el cual también los más experimentados se siguen perfeccionando. De esta manera, los seres humanos nos vamos habilitando a través de nuestros involucramientos en los entornos de vida, fomentando la atención hacia cómo todas las líneas de vida se relacionan y se entretejen en forma continua, sin necesariamente tener una finalidad específica o totalizante. "Atención" es claramente un concepto clave: sugiere una importante variedad de connotaciones prácticas: estar atento a y prestar atención; percibir y así prepararse, adquiriendo un cierto dominio práctico; atender en el sentido de to attend to, también en el sentido de cuidar activamente; y finalmente, a partir del francés attendre o attente, conlleva la idea de la espera, de una atención y un cuidado expandido a través del tiempo, entre la competencia y la vulnerabilidad, entre la sintonización activa y el anhelo paciente. En reflexiones recientes, Tim Ingold asocia esta múltiple acepción de atención/atender metafóricamente con una trenza continua (o braid), y no solo un conjunto o una red cerrada (como una comunidad, un grupo social cerrado, una sociedad) (Ingold 2015). La socialidad o convivencia (siempre en el sentido amplio) para él se va trenzando 'atencionalmente' -y no intencionalmente- a partir del hacer cotidiano, a lo largo de los caminos o las líneas de la vida, sin una necesaria finalidad.

En estudios anteriores y como resultado de un trabajo de campo de larga duración con familias aymara en El Alto (a partir de1995), propuse analizar la 'progresión' indígena y las fuertes mareas y tensiones interculturales que marcaban su tradición cultural a partir de un choque entre dos "intuiciones culturales". Intuiciones en el sentido de "cauces para la acción", internalizados a lo largo del aprendizaje y la cosmopraxis, a través de las generaciones. Por un lado una intuición más bien 'occidental', del distanciar/disociar y atomizar, versus una intuición cultural del contextualizar, que caracterizaría más bien la tradición aymara y que se definió como cierta 'habilidad' (o skill) para relacionar atenta y activamente los diferentes contextos de la vida-en sus dimensiones temporales y espaciales-que permiten la convivencia de los integrantes del mundo (Arnold y Yapita 1992, 1998; De Munter 2007, 2009, 2010). Para la teorización de aquella investigación fue crucial la obra del psicólogo cultural Michael Cole (1996), quien había propuesto, a su vez, romper las clásicas aproximaciones cognitivistas mediante la pista de la "contextualización cultural", dinamizando el conocido término de 'contexto' como una constante práctica de contextualización, en su plena dimensión ecológica y culturalmente relacionadora. De los resultados de aquella investigación doctoral y postdoctoral se destacó que varios informantes/ consejeros aymara -notablemente las mujeressubrayaban que, para que su tradición pudiera seguir "viva", era necesario "practicar la familia", expresión que asociaban con varias prácticas concretas, como el tumpañ $a^{7} \mathrm{o}$ visitar, y con nociones más generales como thakhichaña: hacer(se) camino por la vida, de manera atenta, como aymara, en un sentido muy afín a cómo Ingold usa wayfaring (Ingold 2000). Sobre todo relacionaban el "practicar la familia" con el ayni, concepto que resume actualmente las variadas prácticas de la "reciprocidad" (De Munter et al. 2006). Este 'practicar' se puede concebir como un "hacer familia", la familia que se tiene que "hacer", cotidianamente. Un arte de hacer familia, una praxis familiar a lo largo de la cual se fomenta una constante educación de la atención hacia cómo se relacionan todos los procesos de vida, entre los cuales se trenza justamente la vida social humana -de la que "hacer familia" es una metáfora por excelencia. El making, insiste Ingold, se da siempre en medio de las dinámicas generales 
del growing. Juntos -haciendo/creciendo/cultivando(considerando la interesante ambigüedad del verbo to grow) constituyen la dimensión de orden pasivoactivo que mencionamos supra: por un lado todo que va "creciendo" alrededor nuestro (y también dentro de nosotros), aquello que nos envuelve, y nos guía. Al mismo tiempo, sometido a lo primero, es lo que nosotros podemos cultivar o criar activamente, como parte del estando-vivo. Se trata entonces de volver la educación o la crianza atenta hacia esta vital y ambigua tensión entre dinámicas de crecimiento y procesos de acción y creación.

\section{Visitar y Conmemorar entre los Aymara}

Revisemos como ilustración de la cosmopraxis aymara en general, y del "practicar la familia" en particular, los principales movimientos que caracterizan el conmemorar a los muertos a principios de noviembre, una época conocida en el calendario agrícola y ritual aymara como jallu pacha (tiempo/espacio de lluvia). Se trata de uno de los momentos culminantes de las prácticas del ayni, que constituyen el motor mismo de los ritmos espirálicos de ese calendario ritual-agrícola. De manera general, estas prácticas se dejan comprender muy bien en términos de las "correspondencias" que caracterizan el estando-vivo (como ontología relacional) y permiten observar las maneras o ways de las que los grupos humanos procuran sintonizar con ellas y también entre sí (como "correspondencia humana"). El esbozo de los principales movimientos y flujos de socialidad aymara que se ofrece aquí a fin de contextualizar la reflexión teórica se inspira en varias visitas y observaciones etnográficas intermitentes a una uta (casa + familia) en un barrio en El Alto a lo largo del período 1995-2015. Sus miembros se mueven entre la gran ciudad de El Alto, donde tienen su residencia principal y sus comunidades de origen -en la mayoría de los casos comunidades cercanas al lago Titicaca-. Siguiendo las opciones teóricas del presente artículo, no entraremos aquí en la interpretación simbólica de dichas conmemoraciones, que quedó ampliamente documentada y discutida en estudios como, entre varios otros, Ortega (2001), Van den Bergh (1990) y Van Kessel (1999). Nos limitaremos a esbozar cómo fluyen y se "trenzan" las principales líneas de esta socialidad aymara-andina a través de prácticas conmemorativas que duran varios días. Obviamente también estudios como los recién mencionados, por debajo de sus enfoques semióticos, pueden brindar interesantes informaciones respecto del discurrir de las prácticas (las personas mismas generalmente no se preocupan mayormente por estos significados simbólicos, o simplemente los desconocen). Aun si se pueden presentar diferencias y variaciones menores de región en región, lo que se impone son ciertos ritmos y principios comunes de praxis social. Es la praxis visitante y conmemorativa, a inicios de noviembre, que permite a los miembros del grupo habilitarse de maneras concretas e intensamente sociales en el manejo de y la sensibilidad ante las -acudiendo nuevamente a este concepto central en la obra de Ingold- "correspondencias" vitales que se van dando entre muertos, vivos, cosas e integrantes en general en lo que comúnmente se conoce como pachamama, la "dama de tiempo y espacio", además de importantes entes o presencias del cosmos y del ambiente (inti, phaxsi, waranaka, wayra, etc.). No tiene sentido hablar de diferenciaciones del tipo naturaleza/cultura, ya que, según el paradigma cosmopraxis, no pensamos desde un plano de construcciones cognitivistas que buscan categorizar, distinguir y controlar. Prevalece enfocar el sumergirse y el 'atender', sin discriminar categorías, ya que estas dificultan precisamente ver, pensar y sentir la vida social como parte (participante) de las correspondencias vitales en general. Dificultan ver cómo, según la lectura conceptual de Ingold, más que "interactuar" con el mundo, le "respondemos" a lo largo de los movimientos de nuestra propia "con-ciencia" con los flujos de la vida animada en el "medio ambiente". El con-memorar, en sus dimensiones cotidianas y rituales (cotidianeidad y ritualidad se permean y confunden constantemente), fomenta una conciencia contextualizante muy concreta hacia la relacionalidad del "estando-vivo".

Los movimientos centrales que impulsan las actividades durante estos días, recordando la importancia señalada por nuestras informantes del "practicar la familia" en la progresión de su tradición indígena, se podrían captar mediante un par de "verbos" o verbi-como líneas/ lines o flujos de prácticas. Son verbos que plasman muy bien las prácticas y los movimientos de la socialidad en general y al mismo tiempo traducen los de las conmemoraciones en particular. Estos 'verbos de la socialidad', nos los mencionaron algunas de las mujeres aymara cuando nos hablaban de la importancia del practicar la familia en su tradición 
(De Munter et al. 2006). Uno es el ya mencionado tumpaña o visitar, otro sería ch'allaña-ch'allar-. Ambos son verbos que evocan de manera precisa las dinámicas relacionadoras que revelan, subrayan y crean las múltiples correspondencias de vida. Tumpaña se refiere concretamente a la acción de visitar o visitarse, de casa en casa, de familia en familia, de la ciudad hacia las comunidades en el altiplano, o viceversa. Es la vida social en constante movimiento, entre casas, familias, vivos y muertos, paisajes, comunidades, cerros y lugares de agua. "Visitar"-y todo lo que conlleva- constituye en su intensa cotidianeidad sin duda un tema constante en la antropología cultural y social (Allerton 2012; Ortner 1978; Rosaldo 2006). Entre los aymara, este continuo visitarse, relacionador e intenso, contribuye directamente a lo que Renato Rosaldo en su clásico ensayo sobre el "Visitar entre los Ilongotes" llamó la "gracia social". "Gracia", a su vez, es un concepto clave en la extensa teorización sobre la reciprocidad elaborada por Dominique Temple (Temple et al. 2003), quien reflexiona de manera radical respecto de las consecuencias "sociales" del sistema capitalista, logrando aterrizar sus reflexiones filosóficas y antropológicas dialógicamente en una praxis social-económica aymara basada en complejas -y a veces ambiguas- prácticas de ayni. Esta praxis de la reciprocidad, generadora de "gracia" social y humana en la lectura de Temple y Rosaldo, se opone a los espacios económicos y sociales impulsados por el interés, el prestigio y la posesión individuales, alejados de cualquier concepción ecológica y solidaria de la convivencia. Asimismo, como observa Renato Díaz en su compilación de ensayos de Renato Rosaldo, "Al explorar las posibilidades humanas que ofrece una situación social específica, Renato Rosaldo hojea sus diarios de campo para narrar cómo el visitar, entre los Ilongot, escapa a una definición estructural y, no obstante, goza de una cualidad de apertura, que revela un sentido de improvisación y gracia social culturalmente valorados" (Díaz 2007:35, énfasis mío). El visitar(se) o tumpaña entre los aymara, cotidiano y concreto, desde su abierta potencialidad improvisadora y en su relación directa con la valorada "gracia social", siempre implica apthapiña o "llevar-comida-para-compartir" (en una traducción libre y dinámica). El apthapi es una comida colectiva -tubérculos, choclos-, generalmente transportada en un awayu y que se ofrece y se comparte al llegar a la casa que uno va a visitar.

Pues, en los primeros días de noviembre, cuando se recuerda con especial atención y cariño a los difuntos, este tumpaña-y el apthapiña asociado-se intensifica y refuerza la convivencia entre muertos, vivos y otros integrantes o seres del pacha (tiempo espacio) de maneras intensas y co-participativas. Así, en esos días se cubren todas las dimensiones de la noción de "atención" mencionadas anteriormente: esperar, atender, cuidar, respetar, relacionar. En cuanto al segundo verbo o flujo de prácticas, ch'allaña (literalmente, 'libar'), este también se refiere a actividades comunes, pero como verbum -o line- tiene que ver específicamente con lo que logran realizar -relacionar y contextualizarprácticas sociales más directamente rituales, y esto en múltiples ocasiones: en las casas, el trabajo, los cerros sagrados, el cementerio, etc. Todos lugares y momentos en los cuales y con los cuales importa "educar la atención" hacia esa "socialidad ampliada', recordando instancias anteriores de las diferentes líneas de vida que posibilitaron celebrar aquello que se está haciendo en 'el momento'. En realidad se trata siempre de secuencias de ch'allas, en diferentes momentos, que enlazan entre sí. La antropología andina ofrece ejemplos detallados sobre este ch'allaña, por ejemplo por el término de la construcción del techo de una casa (Arnold y Yapita 1991), respecto de ritos cotidianos de agradecimiento sin intervención de especialistas (De Munter 2007), en las visitas a los uywiri (Choque 2008) o ritos -“mesas"- de sanación mental o física (Fernández Juárez 1997), entre varias otras ocasiones. Estas prácticas en torno al ch'allar llaman y fomentan la atención hacia una ontología relacional -hacia las relaciones y correspondencias que dan vida, de modo que las personas aprendan a situarse en y sintonizarse con estos procesos de vida más amplios-. Estas son, según algunos autores, características importantes del actuar ritual y religioso en general (Pinxten 2010; Staal 1996).

En la breve evocación que sigue, aparte de las entrelazadas prácticas del tumpaña-apthatiña y del ch'allaña, juega como tercer verbum abarcador la práctica del 'recordar' o amtaña. Este tercer 'verbo' -siempre como flujo de praxis social, que se entreteje con las líneas del visitar y del ch'allar-no se tiene que comprender como una actividad que se sitúa en o se promueve primordialmente desde la mente, 
sino que se produce en constante correspondencia con otras prácticas sociales y relacionadoras como el tumpaña -llevar y compartir comida, coca, conversar- y el ch'allar-compartir, narrar, recordar, atender, agradecer, dar/libar y rogar-. El amtaña se presenta solamente en apariencia como más 'abstracto', ya que el recordar, que solemos asociar con los pensamientos y con los sueños ${ }^{8}$, se origina de manera intensa en prácticas y cosas "conmemoradoras" muy concretas: los panes que se hacen, las bebidas alcohólicas que se consumen y que "transportan" a los embriagados a otros tiempos, los ritmos de la música que se (re)crean y que hacen bailar -mover pies con parientes y con la tierra, etc.-. Amtaña tiene mucho que ver también con los tejidos que se colocan cuidadosamente en la base de las mesas conmemorativas y con las hojitas de coca que se akhullikan-que no es un mascar para tragar, sino un 'macerar' lento y atento: la sagrada hoja de coca se comparte, se akhullika junto con los demás y de esta manera se crea un ambiente de encuentro y de respeto, en el que se puede con-versar. La coca, intermediaria viviente, habilita la comunicación y convivencia con los antepasados -con el ајауи o "espíritu" de personas, familias y comunidades-. Recordar es conmemorar, es compartir.

Lo que se presenta a continuación es meramente un 'corte' de una dinámica continua que dura mucho más que los 'dos días' evocados aquí. Se registran los movimientos de la vida social según los tres verbos mencionados, y cómo a través de ellos se vuelve "atenta" la crianza ecosocial en general.

\section{Apxata: Conmemorar, Convidar, Convivir}

Las palabras usadas para denominar algunas prácticas sociales en sí también son prácticas relacionales. Así, el aymara tiene un concepto que 'resume' las prácticas conmemorativas a los muertos en su totalidad: apxata, uno de los varios derivados del verbo apaña o llevar/trasladar. Apxata puede referirse muy concretamente a la mesa ritual que preparan las familias en sus casas -la que luego llevarán al cementerio- y en general a todos los movimientos y prácticas que tienen que ver con el con-memorar: visitar, llevar comidas, convidar, compartir comida, bailar en los cerros (Choque 2009). A veces se usa también el verbo castellanizado "apxatar". Apxata, además, se relaciona semánticamente con el ya mencionado apthapi: una comida comunitaria transportada en un awayu, pero en general también "acopio", "reunión" e incluso "cosecha" (Diccionario Laime 1992), lo que nos procura una interesante asociación entre las prácticas del cultivar alimentos y las prácticas 'propiamente' sociales: una socialidad que de alguna manera se deja 'llevar' (apaña) y criar por estas relaciones de producción y reciprocidad con la tierra9.

En octubre, mediante visitas físicas o comunicaciones virtuales, los parientes se ponen de acuerdo para decidir en qué casa o familia (uta en aymara quiere decir ambas cosas, casa y familia) se van a juntar y para recordar a qué difuntos. No es raro que en una misma mesa se conmemore a dos o tres muertos o 'almitas' simultáneamente. Como quiere la costumbre, se 'atiende' -en el rico significado que le hemos dado previamentea aquellas 'almitas' que no llevan más de tres años muertos, ya que al tercer año las familias se pueden 'despedir' alegremente de ellas y dejan de recordarlos (etimológicamente, llevarlos -casi literalmente- en el corazón) de esa manera tan personalizada y atenta. En los días previos al 1 y 2 de noviembre, los parientes -donde tienen un rol especial mujeres y niños- se juntan para hacer una gran cantidad de pancitos, que son transformados en t'ant'a wawa: literalmente "niñitos de pan", aunque también se representan otros personajes e incluso caballos (Choque 2009) -últimamente, algunas de las mascaritas de yeso que se ponen en los panes están hechas a semejanza de los difuntos-. Estos panes serán incorporados en la mesa ritual (apxata) en las utanaka (familias/casas) y, posteriormente, se llevarán como parte del apthapi que se instalará cerca o encima de la tumba de la persona difunta. Pequeñas creaciones vivas que vienen a acompañar el grupo familiar y que al final de los días del conmemorar terminarán siendo comidas -no sin a veces primero servir como juguetes para los niños-. En el artículo Kneading life: women and the celebration of the dead in the Ecuadorian Andes, Emilia Ferrara analiza cómo las habilidades convivenciales de las mujeres, empezando con la preparación de los 'mágicos' panes (agua-harina-levadura-sal-calorcrecimiento-comida-vida- 'wawas'-reciprocidad) y luego también la elaboración de los platos especiales, juegan un rol central en la intensa celebración de la socialidad extendida que se practica en los primeros días de noviembre. Los hijos las acompañan en esta labor y hacen sus propias masitas. De esta manera se van preparando y habilitando, paulatinamente, 
para incorporar ciertos aspectos muy concretos de su cosmopraxis y de la ontología relacional.

Todavía en la fase preparativa, las familias visitan las ferias -sobre todo la conocida " 16 de Julio"- para comprar los pinkillus, instrumentos de viento considerados adecuados para ser tocados en este tiempo cuando se llama a la lluvia (jallu pacha). La feria en aymara se conoce como qhatu, y, según la interpretación de Yampara, sería por excelencia un lugar de encuentro-taypi- y de prácticas de ayni (Yampara y Temple 2008). En la feria, los parientes también compran las coloridas mascaritas de yeso para transformar los pancitos en t'anta wawa o en animalitos. Asimismo, adquieren grandes cantidades de comida, fruta sobre todo, generalmente proveniente de otras zonas ecológicas, como piña y caña de azúcar de los yungas y también flores de los valles templados. Con todos estos materiales en la mañana del 1 de noviembre se monta una hermosa mesa ritual y conmemorativa en el cuarto principal de la casa elegida, construida sobre la base de unos tejidos pertenecientes a los respectivos muertos que se recuerdan.

\section{Enfocando: 1 y 2 de noviembre}

El primer día, a la uta-amti (“casa-familia que recuerda") la visitan parientes -y compadres- tanto de la ciudad como de comunidades. Al día siguiente, ella se moverá hacia las comunidades de origen del pariente difunto, tejiendo así una intrincada malla de prácticas de reciprocidad o ayni. En la mañana del 1 de noviembre los visitantes llegan desde muy temprano, algunos anunciados, otros sin anunciar. Mientras algunos fabrican la mesa conmemorativa, llena de frutas y pancitos, otros preparan comida desde temprano en la mañana, en el patio de la casa, y a media mañana hombres, mujeres y niños se sirven el primer plato caliente del día, el de la saxra hora. Algunos de los platos preparados -los preferidos por los difuntos recordados-irán a integrar también la mesa ritual, alimentando de esta manera $-\mathrm{y}$ haciendo particularmente presentes y vivos- a los difuntos durante este día. El resto del día se desarrolla básicamente a partir de las sucesivas visitas y lo que ellas conllevan. Los parientes más cercanos a los difuntos, junto con estos últimos, hacen de anfitriones, también si la casa no es directamente su propia casa. Las fronteras de la propiedad se diluyen, prevalece la "gracia" del tumpaña-un verdadero ‘bien común' in-the-making.
Se da la bienvenida de manera hospitalaria a todos los visitantes. Algunos traen dones para la mesa recordatoria y saludan a vivos y a difuntos. A estos últimos les 'rezan', les ofrecen oraciones, en una mezcla de aymara y español. En reciprocidad reciben un plato de comida, un vaso de cerveza o gaseosa. Este dar y recibir sencillo y directo, como un motor básico del ayni o reciprocidad, dura todo el día. El tiempo fluye de otra manera, la música lleva el compás: los hombres tocan e invitan a tocar, se come y se ch'alla con cerveza u otra bebida. Para los jaqinaka o personas aymara, todo lo que se come y todo lo que se reza -las palabras son alimento y transmiten cariño- llega a los difuntos y, a través de ellos, a la tierra o pacha(mama) y puede ser devuelto, algún día -ojalá pronto, con la venida de las lluvias, en la medida requerida-. En resumen, ese primer día de noviembre se educa el "practicar la familia" con plena atención hacia el estando-vivo. Según las personas consultadas, este intenso flujo con-memorativo en tiempos antiguos no paraba y duraba hasta la aurora del día siguiente. Hoy, generalmente hacia la medianoche los integrantes de la familia-casa se acuestan y al alba envuelven todo lo que estaba en la mesa conmemorativa, además de otros alimentos, en grandes bultos. Todo ello, además de los instrumentos de música, se traslada al cementerio de la comunidad ${ }^{10}$ donde vivía el difunto re-cordado -o, en caso de varias almitas "atendidas", la persona que ocupa el lugar central en esta praxis conmemorativa-. Ese segundo día van a visitar a los difuntos al cementerio, al lugar donde están enterrados, un verdadero lugar-endevenir que se puede considerar como un $w a k^{\prime} a^{11}$ portentoso. Es la manera (como way/camino/thakhi) de la que muchas familias alteñas se relacionan con sus parientes en la ciudad, con las chacras que trabajan los parientes que se quedaron a vivir en el campo, con los cerros, y, en ciertos casos, también con el lago Titicaca. Este día 2 de noviembre las líneas del visitarse de las familias se extienden y se trenzan gradualmente con las dinámicas visitadoras de otras familias y con los entornos del lugar, los cerros, el lago. Las respectivas familias se mueven con todos los elementos de las mesas, llevan (aptaña/apxata) todos los víveres como un gran apthapi, esta vez una comida colectiva más 'ritual', más amplia, más atencional que de costumbre, y van a visitar a los difuntos en los cementerios, exponiéndose plenamente a la dimensión ecológica de su cosmopraxis conmemoradora. Allí confluyen 
familias que residen en la ciudad y familias o parientes residentes en las comunidades, aunque siempre hay un intenso flujo entre ambos grupos. Todas las familias llevan instrumentos, algunas contratan grupos de músicos, esto generalmente para las "almitas de tres años", a las que se podrá "despachar" de manera más intensa y festiva, con bailes y borrachera compartida. En cualquiera de los casos, la base para la praxis conmemorativa sigue siendo el "practicar la familia" o hacer familia, que ahora en el cementerio se desenvuelve de manera más extendida y abierta, a lo largo de todo el día. En esto participan plenamente los niños, desde su "legítima periferia" (Lave y Wenger 1991), guiados por los abuelos y padres que cuentan con una gran experticia. Ahí se produce una "comunidad de prácticas": aprendiendo a "hacer familia", tanto los niños-aprendices como sus "maestros" se habilitan socialmente y aprenden a sintonizarse con los antepasados, con los paisajes y sus integrantes, con las líneas de vida. Este sintonizarse, si lo leemos desde la metáfora del aoristo de Ingold, implica que se someten atentamente a los procesos de crecimiento y de muerte, esta última como una "fase" ambigua de una línea de vida, ya que no termina realmente, mientras correspondan con las líneas de sus parientes, mientras se hagan co-presentes, como en estos días de noviembre. Los jaqinaka se someten, se dejan 'envolver' por ese mundo en movimiento o crecimiento constante, pero también actúan en él-hacen familia-, apasionadamente.

Es interesante señalar que los cementerios en las comunidades andinas raras veces se encuentran cerca de las iglesias o de los centros de las aldeas. Se sitúan generalmente afuera, en pleno campo, en el Altiplano, ojalá cerca de algún cerro ${ }^{12}$. El contacto con los cerros, la tierra/suelo y eventualmente con el lago u otros lugares de agua se hace sentir a cada momento. Por los senderos vienen llegando cada vez más personas de la comunidad, muchas familias llegan de la ciudad en auto. Las familias se toman el cementerio, el cementerio se vuelve familia. Con cada visita nueva se vuelve más viviente, bulle de vida. Inicialmente, las celebraciones se ven bastante contenidas: alrededor de cada tumba los hombres y los parientes más cercanos se instalan a comer alrededor de la misma -los platos más ricos se colocan encima de la tumba/la tierra/el cuerpo de la persona difunta. Las mujeres generalmente comen agrupadas al pie de la tumba. A menudo, ciertas personalidades espirituales o políticas de la comunidad-yatiri, jilaqata- vienen a sentarse un rato con la familia a compartir un plato y a conversar con adultos y jóvenes, una forma de darles la bienvenida e incorporarlos al nivel de la comunidad. Se come, se les reza a los muertos, se habla del 'porvenir', se come, se con-memora. Llegan otros parientes a ofrecer su oración y reciben comida -todo lo que se come y reza llega al muerto que está muy presente, y a través de él, a la pachamama-. Esto transcurre durante un par de horas, de manera tranquila y concentrándose alrededor de cada tumba hasta que, hacia el mediodía, sin señal aparente y tampoco en ninguna hora específica, ocurre algo impresionante, de forma espontánea. Aquel flujo bastante contenido de la praxis con-memoradora, trasladada desde las casas al espacio social y ecológico más amplio que es el cementerio y a lo largo del cual los jóvenes van incorporando intensamente los ritmos de la convivencia familiar y comunitaria $-\mathrm{y}$ del estando-vivo en general-, en un momento dado se intensifica, se abre y da lugar a un movimiento complejo, enlazando a todas las familias presentes. Efectivamente, después del mediodía, casi como si se hubiesen puesto de acuerdo, varios risiris (personas que rezan) externos a la familia, sobre todo niños pero también algunos ancianos de la comunidad rural, vienen a ofrecer su oración, los parientes del difunto les agradecen con las consagradas palabras, mil veces repetidas en estos días -"que se reciba tu oración"-y luego les obsequian platos con panes, dulces y frutos. Ahí es cuando, a causa de la intervención de esos risiris que ofrecen y reciben, pero ya sin necesidad alguna de "rezar", todas las personas presentes en el cementerio pasan a entre-obsequiarse febrilmente platos atiborrados con alimentos, de tumba en tumba, de familia en familia. De esta manera, al final de la tarde, cada familia no solamente ha regalado casi todo lo que ha traído al cementerio-taypi, sino también ha recibido a veces más de lo que se ha traído. Todos participan en esta extensa práctica de reciprocidad sencilla y cautivante, que es de una impresionante "gracia" social.

No basta con interpretar aquello meramente como una suerte de puesta en escena de la reciprocidad/ del ayni y menos se trata de usar este ejemplo en el marco de una lectura monolítica o esencialista de lo que sería 'la' oikonomía aymara, en su sentido más amplio. Aquí es donde y cuando muy concretamente se trenzan las líneas de vida entre las respectivas tumbas-que-son-casas-que-son-comedores-que-son- 
familias-que-son-tierra, entre la vida en la ciudad y la vida en el campo, entre las casas construidas con gran esfuerzo en la ciudad y la gran casa/familia que es el Altiplano, cerca del lago, entre chacras y achachilas, entre pasado/presente y futuro, entre muertos y vivos: todas estas categorías se borran en este día, se hacen co-presentes y corresponden, vitalmente. Aquí es donde y cuando se aprende a atender a estas correspondencias, elemento clave para entender el a veces idealizado o ideologizado suma qamaña en términos pedagógicos y concretos.

Hacia el final del día 2 de noviembre, para aquellos casos del apxata "de tercer año", este metafórico "baile de socialidad" se extiende hacia los cerros y se vuelve muy físico, con los parientes bailando, con polleras multicolores moviéndose al son de la música, y el alcohol que todo lo une y funde. Una kacharpaya, pero una "despedida" no definitiva, ya que las líneas de vida de esas almas-de-tres-años continúan o continuarán en las generaciones siguientes, según la lectura que hace Ingold del parentesco.

\section{Reflexiones Finales: Correspondencia Humana}

De lo que se ve y vive en estos dos días, se desprende que los muertos siguen siendo tratados -y muy concretamente visitados- como parientes, se los visita en sus casas/familias y en sus lugares de descanso. Descanso relativo, porque durante los tres primeros años, al menos durante esas semanas a fines de octubre y principios de noviembre, siguen "haciendo(se) camino" (thakhichaña) en medio de ese mundo relacional, junto con los vivos. En algunos casos, estos últimos incluso los van a "buscar" al cementerio en la madrugada del 1 de noviembre ${ }^{13}$, tocando su música favorita y llevándoles flores y comida, para luego "traérselos" a las casas. En resumen, se los cuida -y se los "vigila", según otra arista del significado de tumpaña ${ }^{14}-$, como si formaran plenamente parte todavía de la familia. Aunque la expresión 'como si' es infeliz, ya que claramente todavía participan de ella, "envolviendo" con su presencia a los vivos en esta importante parte de su cosmopraxis, de su "hacer familia". Cabe recordar aquí el conocido lema aymara qhip nayra uñtasa nayraqatar saraña/ "mirando atrás y adelante caminemos hacia adelante" ['adelante' como traducción de nayra, que también significa 'pasado', 'fuente' y 'ojo']. Expresión que plasma bien la práctica-y el aprendizaje- del contextualizar y relacionar en la tradición aymara, al discurrir a través del tiempo-espacio. Esta expresión indica el movimiento espirálico de la muy variada y compleja progresión humana, movimiento en el cual la experiencia del pasado-presente, que los humanos vemos y miramos activamente, ya que se encuentra 'delante', va guiando las futuras 'caminatas'. Esta experiencia, "encarnada" de alguna forma en los muertos durante noviembre, es fundamentalmente relacionadora, lo que se refleja, en la expresión, en el omnipresente verbo saraña, que significa 'ir' o 'caminar' y alude a las dinámicas centrales del 'caminar como jaqi'. La frase indica la fuerte relevancia de los viajes o 'caminatas' que uno va realizando entre todo lo que viene (y venga) 'hacia delante', el devenir (crecimiento) que nos envuelve y que nos vuelve atentos a la relacionalidad del estando-vivo. Al respecto, el verbo thakhichaña/hacer(se) camino "corresponde" bien con el wayfaring, noción central en Ingold que alude al modo del que los seres vivos inhabitamos el mundo, moviendo y habilitándonos continuamente a través de los senderos de vida que se nos van abriendo y que nos vamos haciendo. Los jaqinaka emprenden caminatas en un 'futuro' y un 'presente-pasado' que se encuentran 'detrás y delante de nosotros'. El otro verbo central en el lema citado es uñaña, que quiere decir "ver" o "mirar": un 'ver' concreto que supone un saber manejar $-\mathrm{y}$ dejarse atentamente llevar por- los distintos contextos que constituyen la vida social y ecológica, a lo largo de las diferentes etapas de la vida y también de las sucesivas generaciones. Así, a principios de noviembre, el tumpaña, que viaja entre diferentes casas y cementerios "crea" una fuerte co-presencia entre muertos, vivos y otros integrantes de la vida. Al mismo tiempo, a lo largo de esta praxis visitante y viajante, se fomenta la atención necesaria hacia pachaj jutir sariri, "los tiempos/espacios que vienen y se van" (otra expresión en la que aparece el verbo saraña). Los tiempos del aspirar-y-crear y del esperar-yaceptar. Los tiempos de los que "se fueron" pero que dejaron sus líneas de vida enlazadas con las de quienes siguen caminando. Durante esos dos días ${ }^{15}$ de intensos re-encuentros, las "almas"-líneas de vida 'pasadas' que continúan desenvolviéndose"corresponden" intensamente con los vivos y con los entornos, mediante alimentos y bebidas, mediante las melodías tocadas en los pinkillus, mediante el 
movimiento de polleras de mujeres que bailan con hombres en los cerros, los sombreros que arrojan sombras de seriedad y alegría y contrastan con la luz radiante del sol. La casa-uta es una "familia" que se тиеve, a lo largo de diferentes lugares y momentos o tiempos, desde la uta hacia el cementerio, lugar y tiempo del visitarse por excelencia, y viceversa. El apxata entonces es una praxis relacionadora intensa y extensa en la que participan plenamente menos experimentados y experimentados, vivos y muertos ${ }^{16}$ y en la que se dedican "todos juntos" (taqini ${ }^{17}$ ), entre otras cosas, a redistribuir alimentos y materiales que se lograron producir, "junto con" la tierra. Es un "todos juntos" ampliado que, como ya indicó Mauss, resulta de una fundamental y generalizada reciprocidad de dones -alimentos, música, afectos- que genera aprendizajes para la convivencia latu sensu, en presencia de los cerros que protegen, vigilan y educan, y del lago cuyas aguas anudan tiempos e historias. El apxata muestra muy bien cómo se penetran mutuamente las líneas espirálicas (Ingold hablaría de "remolinos" e incluso de "husos") del ayni, envolviendo a jakinaka y a otros integrantes del entorno social y ecológico. Son líneas de vida que corresponden, along lines of growth and becoming (Ingold 2015:54).

\section{Epílogo: Humaning}

Ahí en el cementerio, compartiendo y conviviendo con todas las otras familias y con otros integrantes del estando-vivo, los seres humanos (se) "humanifican" plenamente, si recurrimos al concepto que Ingold toma prestado del pensador medieval Ramón Llull (homificare) y que relaciona a su vez con la filosofía de Ortega y Gasset. Los seres humanos hacemos lo que hacemos porque somos humanos. Human beings are auto-fabricators, said Ortega. They make or build themselves (Ingold 2015:117). Pero además de esta "auto-fabricación", subraya Ingold, en su conocida y radical crítica al neo-darwinismo: humans also grow: like all living beings they undergo a process of ontogenesis (Ingold 2015:120, énfasis mío). Analizar así el estar-hacer-crecer-y-someterse permite captar lo social $-\mathrm{y}$, por extensión, lo humano- de una manera antropológica más abierta, sensible a concebir la socialidad humana en su plena relacionalidad: la ontogénesis humana-Ingold habla de anthropogenesis- se trenza íntimamente con la ontología relacional, con todos los demás procesos de vida, resultado de una vital co-presencia. Si "creamos nuestra humanidad" constantemente a través de las cosas que hacemos, esto implica una importante responsabilidad colectiva, que se tiene que trabajar desde la inevitable tensión entre dos dinámicas entrelazadas, resumido por Ingold en la expresión making-in-the-growing, en el que el segundo tiene predominancia. In-the-growing se refiere a aquello que "nos va pasando" -que nos hace "crecer"-. Se refiere a la parte de la ontogénesis que nos envuelve, lo que paradójicamente no implica una pasividad (como la imagen del aoristo, supra). La "antropogénesis", como "autofabricación" en medio de y a lo largo de la ontogénesis, en medio de un mundo relacional, es entonces algo que está constantemente in-the-making y, al mismo tiempo, es también un someterse atentamente a-un "dejarse criar por" -los ritmos de la vida, de la muerte y la vida-. El making o "autofabricarse" (humaning en Ingold) en el ejemplo de este artículo es el "hacer familia" en un momento culminante: con-memorando, taqini-todos juntos-, entre niños, adultos y ancianos, visitamos a los seres, tiempos, ambientes y lugares queridos y "somos visitados" por todos ellos: ellos nos envuelven, en su morir y vivir.

Agradecimientos: Si bien este artículo se presenta como el trabajo de un autor, en realidad es el fruto de un prolongado diálogo entre muchas voces, muchos tiempos y lugares. Quiero mencionar aquí solamente a Tim Ingold, Lieve Orye, Gerardo Mora, Sol Martínez y Teófilo Laime. Gracias sobre todo a las familias Choque, Mendoza y Bautista en El Alto que siguen siendo, aun en estos tiempos de poco tumpaña, los grandes inspiradores y facilitadores de estos otros senderos antropológicos, junto con los De Munter Coppia. Un saludo especial para don Casto Choque Choque, fallecido en 2014. 


\section{Referencias Citadas}

Allerton, C. 2012. Making guests, making 'liveliness': the transformative substances and sounds of Manggarai hospitality. Journal of the Royal Anthropological Institute 18:49-62.

Astvaldsson, A. 2000. Las Voces de los wak'a: Fuentes Principales del Poder Político Aymara. Jesús de Machaqa: La Marka Rebelde. vol 4. CIPCA, La Paz.

Berkes, F. 2012. Sacred Ecology. Third edition. Routledge, New York.

Caqueo-Urízar, A., K. De Munter, A. Urzúa y J. Saiz 2014. Entre lo aymara y lo chileno: escala de involucramiento en la cultura aymara (EICA). Una aproximación interdisciplinar a la dimensión aymara en la vivencia intercultural de estudiantes de enseñanza básica del norte de Chile. Chungara Revista de Antropología Chilena 46:423-435.

Chaiklin, S. y J. Lave 2001. Estudiar las Prácticas. Perspectivas sobre Actividad y Contexto. Amorrortu, Madrid.

Choque, C. 2009. Culto a los uywiris. Comunicación ritual en Anchallani. ISEAT/Editorial Mama Huaco, La Paz.

De Munter, K. 2007. Nayra, Ojos al Sur del Presente. Aproximaciones Antropológicas a la Interculturalidad Contemporánea. CEPA-Latinas Editores, Oruro.

De Munter, K. 2010. Tejiendo reciprocidades: John Murra y el contextualizar entre los aymara contemporáneos. Chungara Revista de Antropología Chilena 42:247-255.

De Munter, K. 2016a. Progresiones andinas: las prácticas organizativas en el mundo aymara. En El Buen Vivir, Interculturalidades y Mundialización: Una Mirada desde América Latina, editado por J.C. Skewes y A.M. Haliski. Editora Da Universidade Federal do Paraná, Curitiba, en prensa.

De Munter, K. 2016b. "Educar la atención" en la cosmopraxis aymara. Re-pensar el (con)vivir bien desde una antropología de la vida. En Ecología y Reciprocidad. (Con)vivir bien en contextos andinos, editado por De Munter, K., J. Michaux y G. Pauwels. Plural, La Paz, en prensa.

De Munter, Koen, B. Bautista y R. Choque 2006. Warmi Aymara in Altupata. Creativity and resistance among women in an indigenous city: El Alto (Bolivia). Aprior Magazine (Special issue diciembre 2006), pp. 47-57.

De Munter, K. y N. Note 2009. Cosmopraxis and contextualizing among the contemporary aymara. En Worldviews and Cultures, Philosophical Reflections from an Intercultural Perspective, editado por N. Note, R. Fornet-Betancourt, J. Estermann y D. Aerts, pp. 87-102. Springer Verlag, Heidelberg.

Descola, P. 2014. All too human (still). A comment on Eduardo Kohn's "How Forests Think". Hau: Journal of Ethnographic Theory 4:267-273.

Duranti, A. 2015. The Anthropology of Intentions. Language in a World of Others. University of Cambridge Press, Cambridge.

Fernández Juárez, G. 1997. Entre la Repugnancia y la Seducción. Ofrendas Complejas en los Andes del Sur. Centro de Estudios Regionales Andinos "Bartolomé de las Casas", Cusco.

Fernández Juárez, G. 2006. Apxata de difuntos en el altiplano aymara de Bolivia. Revista Española de Antropología Americana 36:163-180.
Ferraro, E. 2008. Kneading life: women and the celebration of the dead in the Ecuadorian Andes. Journal of the Royal Anthropological Institute, vol. 14, no. 2, pp. 262-277.

Gieser, T. 2008. Embodiment, emotion and empathy. A phenomenological approach to apprenticeship learning. Anthropological Theory 8:299-318.

Guattari, F. 1989. Les Trois Écologies. Éditions Galilée, Paris.

Hastrup, A. 2015 (ed.). Anthropology and Nature. Routledge, London.

Holbraad, M., M. Pedersen y E. Viveiros de Castro 2014. The Politics of Ontology: Anthropological Positions. Fieldsights - Theorizing the Contemporary. Cultural Anthropology Online, January 13, 2014, http://culanth.org/fieldsights/462-the -politics-of-ontology-anthropological-positions.

Ingold, T. 1993. The temporality of the landscape. World Archaeology 25:24-174.

Ingold, T. 1996. Key Debates in Anthropology. Routledge, London.

Ingold, T. 2000. The Perception of Environment: Essays on Livelihood, Dwelling and Skill. Routledge, London.

Ingold, T. 2010. Being Alive. Essays on Movement, Knowledge and Description. Routledge, London.

Ingold, T. 2013. Making. Anthropology, Archaeology, Art and Architecture. Routledge, London.

Ingold, T. 2014 On Human Correspondence. Huxley Memorial Lecture, London.

Ingold, T. 2015 The Life of Lines, Routledge, London.

Ingold, T. y T. Kurttila 2000. Perceiving the environment in Finnish Lapland. Body \& Society, 6(3-4):183-196.

Mamani, M. 2010. Kirkir Warmi: identidad y rol de la mujer aymara en el desarrollo musical del norte chileno. Revista Musical Chilena 64 (213):90-102.

Ortega, M. 2001. Escatología andina: metáforas del alma. Chungar, Revista de Antropología Chilena 33:253-258.

Ortner, S. 1978. Sherpas through their Rituals. Cambridge University Press, Cambridge.

Orye, L. 2011. Worldview as relational notion? Reconsidering the relations between worldviews, science and us from a radical symmetrical anthropology. En Worldviews, Science and Us: Interdisciplinary Perspectives on Worlds, Cultures and Society, editado por D. Aerts y B. D'Hooghe, pp. 129-163. World Scientific, Singapore.

Overing, J. y A. Passes (eds.) 2000. The Anthropology of Love and Anger. The Aesthetics of Conviviality in Native Amazonia. Routledge, London.

Pachaguaya Yujra, P. 2008. La Poética de las Vertientes. Ecofeminismo y Posdesarrollo en Santiago de Huari. UMSAPIEB, La Paz.

Pinxten, R. 2010. The Creation of God (Gods, Humans and Religions). Peter Lang, New York.

Pinxten, R. y K. De Munter 2010. De Culturele Eeuw. Acco, Leuven.

Rosaldo, R. 2006. Visitar entre los ilongotes, gracia social y los ritmos de la vida cotidiana. En Ensayos en Antropología Crítica, 
editado por R. Díaz Cruz y R. Rosaldo, pp. 41-62. Juan Pablos, Fundación Rockefeller, Universidad Autónoma MetropolitanaIztapalapa, México, D.F.

Staal, F. 1993. Rules without Meaning: Ritual, Mantras, and the Human Sciences. Peter Lang Verlag, Berlin y New York.

Temple, D., F. Layme, J. Michaux, M. González y E. Blanco 2003. Las Estructuras Elementales de la Reciprocidad. TariPlural, La Paz.
Van den Berg, H. 1990. La Tierra No Da Así No Más. Los Ritos Agrícolas en la Religión de los Aymaras-Cristianos. Hisbol, La Paz.

Van Kessel, J. 1999. Los Vivos y los Muertos. Duelo y Ritual Mortuorio en los Andes. IECTA, Iquique.

Yampara, S. y D. Temple 2008. Matrices de Civilización. Sobre la Teoría Económica de los Pueblos Andinos. Qamaña PachaQullana Suma, La Paz.

\section{Notas}

1 Una observación introductoria en cuanto al uso y también las traducciones que en este artículo se presentan de los escritos y los conceptos de Tim Ingold. Se trata de una obra teórica importante -aún poco traducida-que establece sus fundamentos a principios de los noventa y que ha ido evolucionando a lo largo del tiempo, afirmando y profundizando un pensamiento antropológico que se puede caracterizar de radical, innovador e integrador, y que ha sido beneficiado por un lenguaje extremadamente cuidado, poético, científicamente agudo y convocador, cuyo alcance, franqueza y sensibilidad no siempre son fáciles de rendir en otro idioma.

2 Al hablar de tradición aymara estamos plenamente conscientes de la gran variedad de contextos (trans)nacionales y situaciones interculturales en la que esta tradición indígena se desenvuelve y se rearticula constantemente. Por debajo de esta diversidad, se puede hablar todavía de una tradición (pan-)aymara, basada en varias costumbres compartidas y a base del idioma aymara, que, aun donde ya no se practica como tal, es una referencia cultural fundamental.

3 Cabe señalar que usamos "praxis", tanto en general como en el uso específico que le damos en "cosmopraxis", de una manera en primera instancia "neutra", como "conjunto dinámico de prácticas". Esto no impide que debiéramos estar abiertos a medir también las dimensión política de "transformación" y de cambio que emana de estas prácticas -sociales, de reciprocidad, etc.-. Esto excede, sin embargo, el alcance directo del presente artículo. Así, por ejemplo, el análisis que aquí proponemos invita claramente a ser relacionado con o traducido en lecturas críticas-pedagógicas de lo que sería el famoso "buen vivir". Esto último se desarrolla en De Munter 2016b.

4 En alusión al libro de Jane Bennett sobre Vibrant Matters. Ingold mismo habla de things, no de objects.

5 Al respecto es interesante el ritual aguatatay en Santiago de Huari, descrito por el antropólogo aymara Pedro Pachaguaya. Se trata de un ritual en el que justamente los niños tienen un rol muy importante cuando cantan para llamar la lluvia. Pachaguaya lo describe como uno de los rituales "donde los seres que componen el paisaje de Huari y las deidades superiores que existen dialogan con los seres humanos" (Pachuaguaya 2008:107).

6 El concepto de cosmo-visión puede sonar algo infeliz por su referencia a "cosmos" en comparación con worldview, pero tiene ventajas ecológicas y holísticas, como en el primer volumen del clásico ensayo Kosmos, de Alexander von Humboldt. Esta dimensión ecológica explica por qué no nos sentimos incómodos con la primera parte del concepto: cosmopraxis (el inglés worldview se podría considerar como más antropocentrado, refiriéndose al mundo de los humanos).

7 Tumpaña es un verbo aymara con un significado "ambivalente", o tal vez mejor, con dos significados complementarios. En este artículo nos referimos a la práctica muy concreta de tumpaña como "visitar(se)", sin embargo, tumpaña también puede tener el significado de vigilar y de sospechar (Diccionario Castellano-Aymara de Félix Layme), lo que permitiría también hacer lecturas más en clave de control social que emana de estas prácticas visitantes. Esto no es de relevancia directa para la presente reflexión sobre la convivencialidad ampliada, en su plena dimensión ecológica y cosmológica, pero claramente es otra arista importante del "hacer comunidad" que podría incluso leerse en términos del enskilment y sobre todo, como una vigilancia que no se limita solamente a lo social-humano.

8 Una forma de saludarse antes bastante común pero todavía en uso es la fórmula "Kunjamakis samkamaxa"/?Cómo te has soñado?”. Información de Teófilo Laime Ajacopa, 2015.

9 Teófilo Laime, lingüista aymara, define apxata como sigue: "apxata es cualquier actividad que los acompañantes llevan algo (merienda, regalo u otra cosa) para generar el ayni. Todo familiar e invitado va con apxata. Esto ocurre en varias fiestas rituales" (comunicación personal Teófilo Laime, 2015).

10 En las últimas dos décadas hay cada vez más familias que entierran a sus muertos en los grandes cementerios en El Alto mismo. Esto no cambia fundamentalmente la dinámica conmemorativa, pero claramente sí implica diferencias en cuanto a los lazos con los parientes en las comunidades y con estas comunidades mismas. La presencia de los achachilas -Illimani, Wayna Potosí y otros- sin embargo se mantiene, aunque cambian los lugares del encuentro y de referencia.

11 Ver Astvaldsson 2000, quien plantea -refiriéndose entre otros a Salomon- que el término "huaca" se empleaba para describir lugares naturales, estructuras construidas y objetos especialmente designados, y destaca la íntima relación de determinadas huacas -o wak'as-con los derechos de aguas, con los fenómenos meteorológicos en general, con los enterratorios y con los muertos, con el origen mítico de los grupos étnicos y, por fin, con un complejo culto a los antepasados.

12 Esto se explica en parte porque tradicionalmente se siente que los muertos de por sí cargan mucha energía (tanto 
"buena"como "mala", según la lógica práctica de lo contradictorio -o del "tercero incluido"- que caracterizaría a la tradición aymara) y una energía que no viene siendo tocada por las creencias católicas, sino una energía del compartir y del transitar, entonces en tiempos precolombinos no se acostumbraba enterrarlos juntos, ya que era mucha energía "junta", sí se solía enterrarlos en los cruces de caminos.

13 Trabajo de campo del autor en El Alto, noviembre 2015.

14 David Choquehuanca en http://servicioskoinonia.org/agenda/ archivo/obra.php?ncodigo $=760$, al referirse al tumpaña dice: "Llamada tumpa en aymara, la responsabilidad de la comunidad es cuidar a sus miembros y su entorno, de manera que cada uno cuidamos la salud y el bienestar de todos y todo sin que falte nadie. Siempre nos preocupamos, nos vigilamos, nos estamos curioseando".

15 El con-memorar no termina el 2 de noviembre, sino que a menudo continúa también los días después.

16 Los difuntos, según ciertas interpretaciones, interrumpirían su "largo viaje" para morar todavía un par de veces -tres vecesde manera más presente con los vivos (Van Kessel 1999).

17 Taqini, literamente: "(entre) todos". Esta categoría implica la relación de las personas con la comunidad buscando un objetivo común, trazado por la misma comunidad y guiado por medio de las autoridades. Esta categoría taqini "todos" es el identificador de la reciprocidad comunitaria, llamada jayma (comunicación personal Teofilo Laime, 2016). 